\title{
A Suzaku observation of the ULIRG IRAS19254-7245: discerning the AGN component
}

\author{
V. Braito ${ }^{1,2}$, J. N. Reeves ${ }^{2,3}$, R. Della Ceca ${ }^{4}$, A. Ptak ${ }^{2,5}$, G. Risaliti ${ }^{6,7}$, and T. Yaqoob ${ }^{2,5}$ \\ ${ }^{1}$ Department of Physics and Astronomy, Leicester University, Leicester LE1 7RH, UK \\ e-mail: bv67@star.le.ac.uk \\ 2 Department of Physics and Astronomy, Johns Hopkins University, Baltimore, MD 21218, USA \\ 3 Astrophysics Group, School of Physical and Geographical Sciences, Keele University, Keele, Staffordshire ST5 5BG, UK \\ 4 INAF - Osservatorio Astronomico di Brera, via Brera 28, 20121 Milan, Italy \\ 5 Astrophysics Science Division, Code 662, NASA/Goddard Space Flight Center, Greenbelt, MD 20771, USA \\ INAF - Osservatorio Astrofisico di Arcetri, Largo E. Fermi 5, 50125 Florence, Italy \\ 7 Harvard-Smithsonian Center for Astrophysics, 60 Garden Street, Cambridge, MA 02138, USA
}

Received 12 December 2008 / Accepted 27 April 2009

\section{ABSTRACT}

\begin{abstract}
We discuss a long Suzaku observation of IRAS 19254-7245 (also known as the Superantennae), one of the brightest and well studied Ultra Luminous Infrared Galaxies in the local Universe.

This long observation provided the first detection of IRAS 19254-7245 above $10 \mathrm{keV}$, and a measurement of a 15-30 keV flux of $\sim 5 \times 10^{-12} \mathrm{erg} \mathrm{cm}^{-2} \mathrm{~s}^{-1}$. The detection above $10 \mathrm{keV}$ has allowed us to determine, for the first time, the intrinsic luminosity of the AGN hosted in IRAS 19254-7245, which is strongly absorbed $\left(N_{\mathrm{H}} \sim 3 \times 10^{24} \mathrm{~cm}^{-2}\right)$ and has an intrinsic luminosity $(L(2-10 \mathrm{keV})$ $\sim 3 \times 10^{44} \mathrm{erg} \mathrm{s}^{-1}$ ) in the QSO regime.

The $2-10 \mathrm{keV}$ spectrum of IRAS $19254-7245$ is remarkably hard $(\Gamma \sim 1.2)$, and presents a strong iron line $(E W \sim 0.7 \mathrm{keV})$, clearly suggesting that below $10 \mathrm{keV}$ we observe only reprocessed radiation. Since the energy of the $\mathrm{Fe} \mathrm{K}$ emission is found to be $\sim 6.7 \mathrm{keV}$, consistent with He-like Fe, and its EW is too high to be explained by a starburst-dominated scenario, we suggest that the $2-10 \mathrm{keV}$ emission of IRAS 19254-7245 is dominated by reflection/scattering from highly ionized matter. Within this latter scenario, we found that the photon index of the illuminating source is $\Gamma=1.87_{-0.28}^{+0.11}$, in excellent agreement with the mean value found for radio quiet unobscured AGN.
\end{abstract}

Key words. galaxies: active - galaxies: individual: IRAS 19254-7245- galaxies: Seyfert - X-rays: galaxies

\section{Introduction}

Ultra luminous infrared galaxies (hereafter ULIRGs) are an enigmatic class of sources which emit most of their energy in the far-infrared (FIR, 8-1000 $\mu \mathrm{m}$ ) domain (Sanders \& Mirabel 1996), with luminosities above $\sim 10^{12} L_{\odot}$, (i.e., comparable to QSO luminosities). The importance of understanding the physical processes at work in ULIRGs is strengthened by the observational evidence that they are generally advanced mergers of gasrich galaxies; these events are now considered to be at the origin of some of the massive elliptical and S0 galaxies (Hopkins et al. 2005, 2006; Springel et al. 2005) and the QSO stage could be a phase in the evolution of these systems. However, understanding their physical nature is complicated by the large amount of obscuration from dust present in these sources, which makes it difficult to directly observe the nuclear source.

X-ray observations of ULIRGs performed with XMM-Newton (Franceschini et al. 2003; Braito et al. 2003), Chandra (Ptak et al. 2003; Teng et al. 2005), and more recently Suzaku (Teng et al. 2009) have proved to be a fundamental tool in distinguishing between the contributions of starburst and AGN activity and to investigate the presence of hidden AGNs in these sources. These observations have shown that ULIRGs are intrinsically faint $\mathrm{X}$-ray sources, their observed X-ray luminosities being typically $L(2-10 \mathrm{keV}) \lesssim 10^{42}-10^{43} \mathrm{erg} \mathrm{s}^{-1}$. The X-ray spectra of ULIRGs are complex and exhibit characteristics of both starburst and AGN activity, confirming the composite nature of ULIRGs. These studies have also shown that more than half of the local brightest AGN-ULIRGs (5/8) host an obscured AGN, with three being Comption Thick $\left(N_{\mathrm{H}}>10^{24} \mathrm{~cm}^{-2}\right.$; NGC6240, Vignati et al. 1999; Mrk 231, Braito et al. 2004; UGC5101, Imanishi et al. 2003). Observations above $10 \mathrm{keV}$ are thus fundamental for measuring the intrinsic X-ray luminosity of obscured AGN hosted in ULIRGs and its contribution to their high observed FIR emission.

IRAS 19254-7245 (also known as the SuperAntennae) belongs to a flux limited sample at $60 \mu \mathrm{m}$ consisting of the 15 brightest nearby ULIRGs (Genzel et al. 1998). Located at $z=0.062$, IRAS $19254-7245$ has an infrared luminosity of $L_{8-1000 \mu \mathrm{m}}=1.1 \times 10^{12} L_{\odot}$ corresponding to a bolometric luminosity of $L_{\text {bol }} \sim 4 \times 10^{45} \mathrm{erg} \mathrm{s}^{-1}$. Like most ULIRGs, IRAS 19254-7245 is a merger system of two gas-rich spiral galaxies.

The southern nucleus, optically classified as a Seyfert 2 , is one of the brightest nearby ULIRGs, which has been found to host both a powerful starburst and an obscured AGN, while there is no evidence of AGN activity in the northern nucleus.

A previous X-ray observation of IRAS 19254-7245 performed with XMM-Newton suggested that this ULIRG harbors a heavily obscured and high-luminosity AGN. The hard powerlaw continuum above $2 \mathrm{keV}$ (photon index $\Gamma=1.3$ ) and the detection of a strong $\mathrm{Fe}-\mathrm{K} \alpha$ emission line at $6.5 \pm 0.1 \mathrm{keV}$ 
$(E W \sim 1.4 \mathrm{keV})$ were indeed highly indicative of a Comptonthick source (Braito et al. 2003). Since the two nuclei are located only $\sim 9$ arcsec apart from each other, XMM-Newton was unable to resolve them; however, the centroid of the hard X-ray emission was found to be spatially coincident with the southern nucleus. A Chandra observation that could resolve this issue has not yet been performed.

The best-fit model obtained for the $0.5-10 \mathrm{keV}$ X-ray emission detected with XMM-Newton consisted of by a strong soft thermal component associated with starburst emission and a hard $\mathrm{X}$-ray component associated with AGN activity. This AGN component was parametrized by a Compton thick AGN model, and consisted of a pure Compton-reflected continuum (with $\Gamma \sim 1.8$ ), a scattered power law component and a strong Fe emission line. The observed 2-10 keV luminosity of the AGN was found to be $\sim 4 \times 10^{42} \mathrm{erg} \mathrm{s}^{-1}$. Due to the limited energy bandpass of XMMNewton, this observation did not allow us to directly see the intrinsic continuum, and thus to measure the absorbing column density and the intrinsic X-ray luminosity of IRAS 19254-7245.

Here we present the analysis of a deep Suzaku observation ( $\sim 150 \mathrm{ks})$ of this system, which allowed us for the first time to constrain the intrinsic power of the AGN hosted by IRAS 192547245 , as well as to investigate in detail the properties of the $\mathrm{Fe}$ line complex. In Sect. 2 we present the Suzaku data analysis and results, while in Sect. 3 we discuss the overall scenario for X-ray emission of IRAS 19254-7245. Throughout this paper, the current standard cosmology is assumed with $H_{0}=$ $73 \mathrm{~km} \mathrm{~s}^{-1} \mathrm{Mpc}^{-1}, \Omega_{\mathrm{M}}=0.27$, and $\Omega_{\lambda}=0.73$.

\section{Observations and data reduction}

Suzaku (Mitsuda et al. 2007) is the fifth Japanese X-ray satellite, which carries on board four sets of X-ray mirrors, with a X-ray CCD (XIS; three front-illuminated, FI, and one backilluminated, BI Koyama et al. 2007) at their focal plane, and a non imaging hard X-ray detector (HXD, Takahashi et al. 2007). The latter consist of 2 main instruments: the Si PIN photodiodes and the GSO scintillator counter. Altogether, the XIS and the HXD-PIN cover the $0.5-10 \mathrm{keV}$ and $12-70 \mathrm{keV}$ bands, respectively.

Suzaku observed IRAS19254-7245 for a total exposure time of about $150 \mathrm{ksec}$; the observation was performed at the beginning of November 2005, when all 4 XIS were still working ${ }^{1}$.

Cleaned event files from the version 2 of the Suzaku pipeline processing were used with the standard screening ${ }^{2}$. The net exposure times are $97.9 \mathrm{ks}$ for each of the XIS and $142.1 \mathrm{ks}$ for the HXD-PIN. The XIS source spectra were extracted from a circular region of $2.9^{\prime}$ radius (which correspond to an energy encircled fraction of $90 \%{ }^{3}$ ) centered on the source. Background spectra were extracted from two circular regions of $2.4^{\prime}$ radius

\footnotetext{
1 Later that month Suzaku XIS2 failed. No charge injection (see: http://suzaku.gsfc.nasa.gov/docs/suzaku/analysis/ sci.html) was applied at the time of the observation so the nominal energy resolution of the XIS at $6 \mathrm{keV}$ was degraded with respect to the prelaunch one

2 The screening filter all events within the South Atlantic Anomaly (SAA) as well as with an Earth elevation angle (ELV) $<5^{\circ}$ and Earth day-time elevation angles (DYE_ELV) less than $20^{\circ}$. Furthermore also data within $256 \mathrm{~s}$ of the SAA were excluded from the XIS and within 500 s of the SAA for the HXD. Cut-off rigidity (COR) criteria of $>8 \mathrm{GV}$ for the HXD data and $>6 \mathrm{GV}$ for the XIS were used.

3 see ftp://legacy.gsfc.nasa.gov/suzaku/doc/xrt/ suzakumemo-2008-04.pdf
}

offset from the source and the calibration sources. Using the latest calibration files available, the XIS response (rmfs) and ancillary response (arfs) files were produced with the ftools tasks xisrmfgen and xissimarfgen, respectively. The net $0.5-10 \mathrm{keV}$ count rates were: $(1.67 \pm 0.07) \times 10^{-2} \mathrm{cts} / \mathrm{s},(1.57 \pm 0.06) \times 10^{-2} \mathrm{cts} / \mathrm{s}$, $(1.55 \pm 0.06) \times 10^{-2} \mathrm{cts} / \mathrm{s}$ and $(1.84 \pm 0.09) \times 10^{-2} \mathrm{cts} / \mathrm{s}$ for the XIS0, XIS2, XIS3, and XIS1 respectively. The source spectra from the three FI CCDs were then combined, while the BI (the XIS1) spectrum was kept separate and fitted simultaneously. The net XIS source spectra were then binned with a minimum $S / N$ of 4 in each energy bin and $\chi^{2}$ statistics have been used.

\subsection{HXD-PIN data reduction}

For the HXD-PIN data reduction and analysis, we followed the latest Suzaku data reduction guide (the ABC guide Version 2) ${ }^{4}$. For the analysis, we used the rev2 data, which include all 4 cluster units, and the backgrounds available (Fukazawa et al. 2009), which account for the instrumental background (NXB; Takahashi et al. 2007; Kokubun et al. 2007). We then simulated a spectrum for the cosmic X-ray background counts (Boldt 1987; Gruber et al. 1999) and added this to the instrumental background.

At the time of writing, two different instrumental background files have been released (background A or "quick" background, and the background D or "tuned" background; Mizuno et al. 2008 , and Fukazawa et al. 2009). We tested both the instrumental backgrounds and we included a $\pm 10 \%$ uncertainty in the level of the CXB. The inspection of the IRAS 19254-7245 net spectrum shows that the source is detected in the $15-30 \mathrm{keV}$ band with both the two background files. The net count rate in the $15-30 \mathrm{keV}$ using background $\mathrm{A}$ and $\mathrm{D}$ are respectively $1.59 \pm 0.14 \times 10^{-2} \mathrm{cts} \mathrm{s}^{-1}$ and $1.48 \pm 0.14 \times 10^{-2} \mathrm{cts} \mathrm{s}^{-1}$, and the corresponding background count rates are $0.25 \pm 0.004 \mathrm{cts} \mathrm{s}^{-1}$ and $0.26 \pm 0.003 \mathrm{cts} \mathrm{s}^{-1}$.

We decided to use the latest release (background $\mathrm{D}$ ), which is affected by lower systematic uncertainties (of about $1.3 \%$ at $1 \sigma$ ), which correspond to about half of the first release of the NXB. Using this background, IRAS 19254-7245 is detected in the 15$30 \mathrm{keV}$ band at $\sim 5.5 \%$ above the background (a total of $\sim 2000$ net counts were collected), corresponding to a signal-to noise ratio $S / N \simeq 10.8$. The dominant component in the background is the instrumental one with a count rate of $0.24 \pm 0.001 \mathrm{cts} \mathrm{s}^{-1}$, while the CXB count rate ranges from $1.4 \times 10^{-2}$ to $1.6 \times 10^{-2}$ when we include the $\pm 10 \%$ uncertainty in its level. If we assume a $10 \%$ higher CXB level, the source is still detected at $5.0 \%$ above the background (its mean count rate in the $15-30 \mathrm{keV}$ is $1.35 \pm 0.14 \times 10^{-2}$ cts s$^{-1}$ ) with $S / N \simeq 9.7$, thus the detection of IRAS 19254-7245 is not dependent on the CXB absolute level. As a further check of the level of the CXB, we analysed the Suzaku observation of the Lockman Hole performed in May 2007. We performed an identical analysis of the CXB HXDPIN observation as we completed for IRAS 19254-7245. The flux of the CXB measured with the Lockman Hole observation was $F(15-50 \mathrm{keV})=1.1 \pm 0.2 \times 10^{-11} \mathrm{erg} \mathrm{cm}^{-2} \mathrm{~s}^{-1}$ (corresponding to a flux density of $\left.3 \times 10^{-11} \mathrm{erg} \mathrm{cm}^{-2} \mathrm{~s}^{-1} \mathrm{deg}^{-2}\right)$, in agreement with the flux of the simulated CXB $(F(15-50 \mathrm{keV})=$ $\left.1.0 \pm 0.2 \times 10^{-11} \mathrm{erg} \mathrm{cm}^{-2} \mathrm{~s}^{-1}\right)$ and the flux measured with both BeppoSAX (Frontera et al. 2007) and Swift (Moretti et al. 2009).

\footnotetext{
4 http://heasarc.gsfc.nasa.gov/docs/suzaku/analysis/ abc/

5 http://www .astro.isas.jaxa.jp/suzaku/doc/suzakumemo/ suzakumemo-2008-03.pdf
} 
Since the HXD-PIN is a non-imaging detector, and taking into account the large field of view of the instrument $(0.56 \mathrm{deg} \times$ $0.56 \mathrm{deg}$ ), we checked that the detection is not caused by to another X-ray source. In particular, we searched the NED database for known AGN in the HXD field of view and inspected the available XMM-Newton observation. We found that two X-ray sources with a 2-10 keV flux comparable to IRAS 19254-7245's emission were detected with XMM-Newton. The two sources are both AGNs, belonging to the XMM Bright Survey sample (XBS J193138.9-725115 and XBS J193248.8-723355; Della Ceca et al. 2004; Caccianiga et al. 2008). XBS J193138.9725115 is a Type $1 \mathrm{AGN}(z=0.701)$ and its XMM-Newton spectrum is well modeled by a single unabsorbed power law component $(\Gamma \sim 2)$ with no evidence of absorption. XBS J193248.8-723355 is a Seyfert 2 at $z=0.287$; the X-ray emission of this source agrees with its classification as a ComptonThin Seyfert; indeed a low energy cut-off is present in the XMMNewton spectra corresponding to $N_{\mathrm{H}} \sim 10^{22} \mathrm{~cm}^{-2}$ and there is no evidence that this source could be Compton thick. The predicted $15-30 \mathrm{keV}$ emission from these sources (derived from the analysis of the XMM-Newton and Suzaku data) is less than $\sim 10^{-13} \mathrm{erg}$ $\mathrm{cm}^{-2} \mathrm{~s}^{-1}$, which is below the HXD-PIN sensitivity and a factor of $\sim 50$ below the measured $15-30 \mathrm{keV}$ flux.

For the spectral analysis, we rebinned the HXD-PIN spectrum of IRAS 19254-7245 to have a signal-to-noise ratio of 5 in each energy bin. To derive a first estimate of the $15-30 \mathrm{keV}$ flux and luminosity of IRAS 19254-7245, we fitted the HXDPIN spectrum assuming a power law model with $\Gamma=1.9$ (i.e., a standard AGN value; Reeves \& Turner 2000; Page et al. 2004; Caccianiga et al. 2004). Taking into account the systematic uncertainties in the NXB model, with this simple model we obtained $F(15-30 \mathrm{keV}) \sim 5.2 \pm 1.1 \times 10^{-12} \mathrm{erg} \mathrm{cm}^{-2} \mathrm{~s}^{-1}$ and $L(15-$ $30 \mathrm{keV}) \sim 4.7 \times 10^{43} \mathrm{erg} \mathrm{s}^{-1}$. The extrapolation of this model down to $2 \mathrm{keV}$ predicts an intrinsic luminosity (which does not include a correction for Compton scattering) of the AGN in IRAS $19254-7245$ of $L(2-10 \mathrm{keV}) \sim 9.5 \times 10^{43} \mathrm{erg} \mathrm{s}^{-1}$.

\subsection{The broad-band spectrum}

Overall the Suzaku observation confirms the XMM-Newton results. A good fit to the $0.5-10 \mathrm{keV}$ Suzaku data is obtained with a model consinting of: a thermal emission component $\left(k T=0.64 \pm 0.10 \mathrm{keV}\right.$ and abundance $Z=Z_{\odot}$, likely associated with starburst activity), a strong hard power-law component ( $\Gamma=1.2 \pm 0.1$, likely associated with AGN emission), and a strong iron $\mathrm{K}$ emission line $\left(E W=710_{-170}^{+190} \mathrm{eV}\right.$, with respect to the observed continuum). The flux and observed luminosity $F(2-10 \mathrm{keV}) \sim 2.9 \times 10^{-13} \mathrm{erg} \mathrm{cm}^{-2} \mathrm{~s}^{-1}$, and $L(2-10 \mathrm{keV})$ $\sim 2.5 \times 10^{42} \mathrm{erg} \mathrm{s}^{-1}$ are found to be consistent with the values measured with XMM-Newton. The de-absorbed luminosity of the starburst component is $L(0.5-2 \mathrm{keV}) \sim 4 \times 10^{41} \mathrm{erg} \mathrm{s}^{-1}$ also in agreement with the luminosity measured by XMM-Newton.

We then tested the best-fit model obtained for the XMMNewton spectrum. In this model, the soft X-ray emission is still modeled with a thermal component, while the hard $(\Gamma \sim 1.2)$ power law component is replaced by a pure Compton-reflected continuum (the PEXRAV model in Xspec, Magdziarz \& Zdziarski 1995, with an intrinsic $\Gamma=1.8$ ) combined with a moderately absorbed $\left(N_{\mathrm{H}} \sim 5 \times 10^{21} \mathrm{~cm}^{-2}\right)$ power law component with the same $\Gamma$, representing the possible scattered emission. The parameters of the reflection component are: an inclination angle $i=45^{\circ}$, abundance $Z=Z_{\odot}$ (using the abundances of Wilms et al. 2000), and a reflection fraction (defined by the subtending solid angle of the reflector $R=\Omega / 2 \pi$ ) $R$ fixed to be 1 .

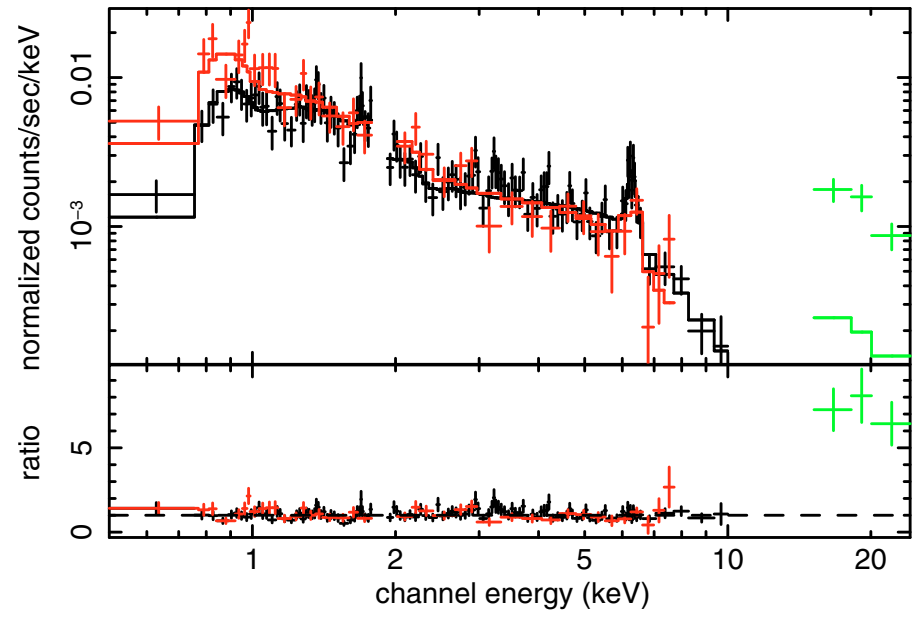

Fig. 1. $0.5-30 \mathrm{keV}$ Suzaku XIS-FI (black data points), XIS-BI (red), and HXD-PIN (green) data and their ratio to the $0.5-10 \mathrm{keV}$ best-fit model. The model is composed by: a thermal emission component dominating the $0.5-2 \mathrm{keV}$ emission, a Compton-reflected continuum $(R=1$, $\Gamma=1.8)$ and a strong Fe emission line at $6.7 \mathrm{keV}(E W \sim 700 \mathrm{eV})$. A clear excess is present above $10 \mathrm{keV}$, which we attribute to the intrinsic X-ray emission of IRAS 19254-7245 transmitted through the high column density absorber.

The normalization of this component was allowed to vary. This model is a good fit to the XIS data alone $\left(\chi^{2} /\right.$ d.o.f. $=159 / 144$, see Fig. 1) and the fluxes and observed luminosity of the AGN components are consistent with the values measured with the XMM-Newton observation. However, this model clearly underpredicts the counts detected above $10 \mathrm{keV}$ (see Fig. 1, green data points). When we include in the fit the HXD-PIN data, fixing the cross-normalization between the XIS and the PIN to 1.16 (Manabu et al. 2007; Maeda et al. 2008 ${ }^{6}$ ), the model is statistically unacceptable $\left(\chi^{2} /\right.$ d.o.f. $\left.=231 / 147\right)$ and even allowing for a harder photon index $(\Gamma \sim 1.3)$ it is not a good representation of the $0.5-30 \mathrm{keV}$ emission $\left(\chi^{2} /\right.$ d.o.f. $\left.=220 / 146\right)$.

To account for the excess detected above $10 \mathrm{keV}$, we added to the model a second heavily absorbed power law component. Since the HXD/PIN residuals suggest the presence of a high column density absorber, we used for this component the model by Yaqoob (1997) (PLCABS in Xspec), which correctly takes into account Compton down-scattering. For high column densities the observed X-ray continuum may also be suppressed by Compton down-scattering, and the intrinsic luminosity must be corrected by a factor $e^{\tau}$, where $\tau=N_{\mathrm{H}} \sigma_{\tau}$ and $\sigma_{\tau}=6.65 \times 10^{-25} \mathrm{~cm}^{2}$ is the Thomson cross-section.

This model provides a good fit to the $0.5-30 \mathrm{keV}$ spectrum $\left(\chi^{2} /\right.$ d.o.f. $=164 / 145$; see Fig. 2 upper panel and Table 1 model A). However, we found a low value of the reflection fraction with respect to this primary absorbed power law component $(R<0.1)$. This suggests that the line and the hard 2-10 keV spectrum are unlikely to be produced by reflection off cold material. The broad band continuum could also be reproduced by a model without the reflected component (see Table 1 model B). Statistically, this model provides a slightly worse fit $\left(\chi^{2} /\right.$ d.o.f. $\left.=181 / 146\right)$ than the previous one, but it is

\footnotetext{
6 http://www.astro.isas.jaxa.jp/suzaku/doc/suzakumemo/ suzakumemo-2007-11.pdf;

http://www . astro.isas. jaxa.jp/suzaku/doc/suzakumemo/ suzakumemo-2008-06.pdf
} 
Table 1. Results of the spectral fit.

\begin{tabular}{ccccccccc}
\hline \hline Model & $\Gamma$ & $\begin{array}{c}N_{\mathrm{H}} \\
10^{24} \mathrm{~cm}^{-2}\end{array}$ & $\begin{array}{c}E_{\mathrm{c}} / \xi \\
\mathrm{keV} / \mathrm{erg} \mathrm{cm} \mathrm{s}^{-1}\end{array}$ & $\begin{array}{c}\sigma^{a} \\
\mathrm{keV}\end{array}$ & $\begin{array}{c}E W^{b} \\
\mathrm{keV}\end{array}$ & $\begin{array}{c}L(2-10 \mathrm{keV})^{c} \\
10^{44} \mathrm{erg} \mathrm{s}^{-1}\end{array}$ & $\begin{array}{c}L(10-30 \mathrm{keV})^{c} \\
10^{44} \mathrm{erg} \mathrm{s}^{-1}\end{array}$ & $\chi^{2} /$ d.o.f. \\
\hline $\mathrm{A}$ & $1.8^{f}$ & $4.2_{-0.9}^{+2.7}$ & $6.67_{-0.05}^{+0.05}$ & $0.11_{-0.06}^{+0.06}$ & $0.60_{-0.22}^{+0.12}$ & 4.0 & 3.6 & $164 / 145$ \\
$\mathrm{~B}^{d}$ & $1.8^{f}$ & $3.1_{-0.4}^{+1.2}$ & $6.66_{-0.05}^{+0.04}$ & $0.14_{-0.06}^{+0.07}$ & $0.86_{-0.25}^{+0.24}$ & 2.1 & 1.9 & $181 / 146$ \\
$\mathrm{C}^{d}$ & $1.20_{-0.04}^{+0.11}$ & $4.1_{-1.3}^{+1.3}$ & $6.67_{-0.04}^{+0.05}$ & $0.12_{-0.05}^{+0.06}$ & $0.67_{-0.15}^{+0.16}$ & 1.7 & 3.9 & $154 / 145$ \\
$\mathrm{D}^{d}$ & $1.87_{-0.28}^{+0.11}$ & $3.2_{-0.5}^{+1.3}$ & $1000_{-430}^{+170}$ & - & - & 2.6 & 2.2 & $199 / 147$ \\
\hline
\end{tabular}

${ }^{a}$ The values of $\sigma$ are the measured ones, which are not corrected for width of the calibration lines, $\sigma_{\text {lamp. }}{ }^{b}$ The $E W$ is measured against the total observed continuum. ${ }^{c}$ The luminosities are derived from the XIS front illuminated CCDs. ${ }^{d}$ These models do not include a cold reflected component.
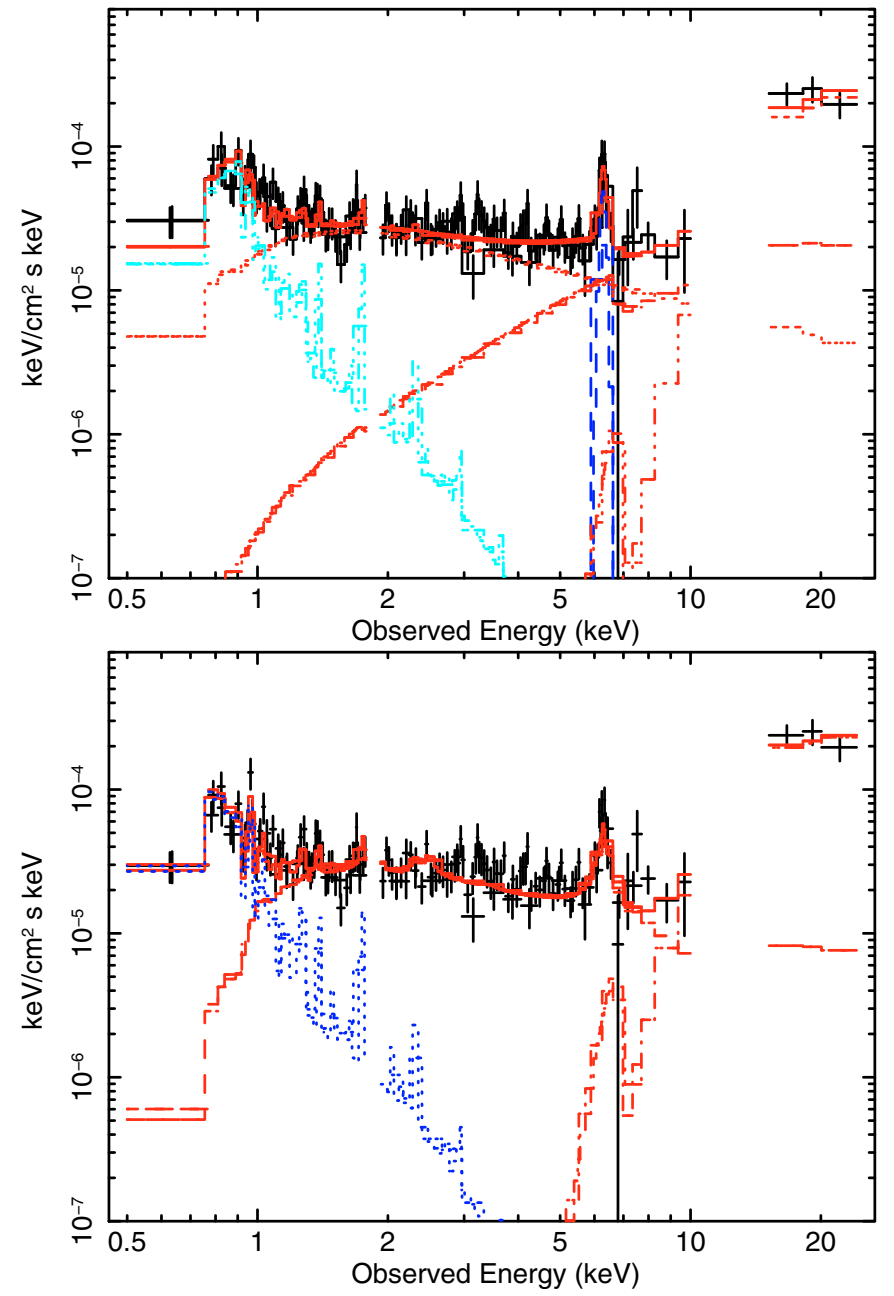

Fig. 2. Upper panel: Suzaku XIS and HXD spectra of IRAS 192547245 where the underlying AGN continuum is modelled with a neutral Compton-reflected component and power-law component seen in transmission through a high column density absorber $\left(N_{\mathrm{H}} \sim 4 \times 10^{24} \mathrm{~cm}^{-2}\right)$. Lower panel: same as above but with the $2-10 \mathrm{keV}$ AGN emission modelled with an ionized reflected component (the high energy component is modelled as in the upper panel with a power-law component seen in transmission through the high column density absorber).

unable to account for the hardness of the 2-10 keV emission. In particular, clear residuals are present in the $5-10 \mathrm{keV}$ band where the reflected component dominates (see Fig. 2 top panel). Finally, if we allowed the photon index to vary, we found that although the fit improves we again need an unusually hard photon index of the power law component $\left(\Gamma=1.20_{-0.04}^{+0.11}\right.$, $N_{\mathrm{H}}=4 \pm 1 \times 10^{24} \mathrm{~cm}^{-2}$; see Table 1 model C).
One of the main results of this observation is that, although we can confirm the presence of a strong Fe line as detected with $X M M-N e w t o n$, the centroid of this line is now at $6.67 \pm 0.05 \mathrm{keV}$ in the rest frame $(E W \sim 0.7 \mathrm{keV}$; for the model without the reflected component), consistent with He-like Fe. Furthermore, the line appears to be marginally broad $(\sigma=0.12 \pm 0.06 \mathrm{keV})$. The inclusion of the line in the model improves the fit by $\Delta \chi^{2}=51$ for 3 degree of freedom ${ }^{7}$. However, if we constrain the line to be unresolved the fit is worse only by $\Delta \chi^{2}=5$. To check the energy and the intrinsic width of the Fe line detected in the XIS, we examined the spectra of the ${ }^{55} \mathrm{Fe}$ calibration source lines, which are located at two corners of each XIS. The calibration source produces lines from $\mathrm{Mn} \mathrm{K} \alpha_{1}$ at $5.899 \mathrm{keV}$ and $\mathrm{Mn} \mathrm{K} \alpha_{2}$ at $5.888 \mathrm{keV}$. From the spectrum of the calibration source, we found that the line energy is shifted redwards by about $25 \mathrm{eV}$, while the residual width is $\sigma \sim 50 \mathrm{eV}^{8}$, confirming that the broadening of the line is intrinsic to the source and not instrumental ${ }^{9}$. After the subtraction in quadrature of this residual width, we obtained $\sigma_{\text {int }}^{2}=110 \pm 60 \mathrm{eV}$ ). However, taking into account the present count statistics of the data, this broadening could be due to the presence of other line components, which are not resolved. In particular, the line profile can be explained with three unresolved Gaussian lines (at $6.4 \mathrm{keV}$, $6.7 \mathrm{keV}$, and $6.96 \mathrm{keV}$; see Fig. 3). Statistically, this model provides a similarly good fit as models with a single broad line $\left(\chi^{2} /\right.$ d.o.f. $\left.=161 / 145\right)$, the strongest line being the $6.7 \mathrm{keV}$ line $(E W \sim 400 \mathrm{eV})$. Although the other two lines are not statistically required, a weak $(E W<200 \mathrm{eV})$ emission line could be present at the energy of the neutral $\mathrm{Fe} \mathrm{K} \alpha$, while the $90 \%$ upper limit to the $6.96 \mathrm{keV}$ line is $150 \mathrm{eV}$.

Since the energy centroid of the line detected by Suzaku appears to disagree with the XMM-Newton results and the $E W$ appears to be lower, we reanalyzed the XMM-Newton data and compared them with the Suzaku results. The exposure time in the XMM-Newton observation was only $20 \mathrm{ks}$, and after taking into account the errors in the flux and line continuum, we found that the two lines are consistent with each other. Furthermore, the energy centroids are consistent to within the errors $\left(E_{\mathrm{XMM}}=\right.$ $\left.6.5 \pm 0.1 \mathrm{keV} ; E_{\text {Suzaku }}=6.66 \pm 0.05 \mathrm{keV}\right)$. Finally, a possible blending of 3 lines was also present in the XMM-Newton data, but again the low exposure time of this observation did not allow a more detailed analysis of the Fe line profile.

\footnotetext{
7 for the scenario with the reflected component the fit improves by $\Delta \chi^{2}=40$.

8 This residual width is due to the degradation of the XIS after the launch and prior to the correction with the charge injection.

9 The intrinsic width of the Fe line in the spectrum of IRAS 192547245 can be $\sigma_{\text {int }}^{2}=\sigma_{\text {meas }}^{2}-\sigma_{\text {lamp }}^{2}$ (where $\sigma_{\text {meas }}$ is the measured width and $\sigma_{\text {lamp }}$ is the width of the calibration lines)
} 


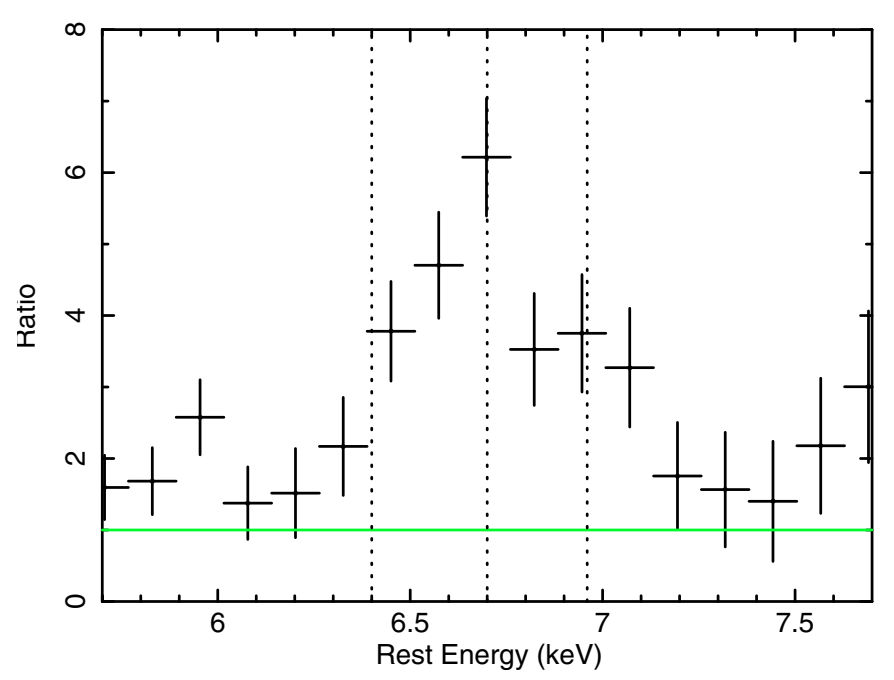

Fig. 3. Residuals of the data/model of IRAS 19254-7245 XIS data at the Fe band, when no iron line is included in the model. The three vertical lines indicate the energy centroids of the three possible components of the Fe line complex $(6.4 \mathrm{keV}, 6.7 \mathrm{keV}$ and $6.97 \mathrm{keV})$. The energy scale is in the rest frame.

From a statistical point of view, all these models are a good representation of the $0.5-30 \mathrm{keV}$ emission, but they are unable to account for the hardness of the continuum. Furthermore, the line energy of the strongest emission line is at odds with a scenario where the $2-10 \mathrm{keV}$ emission is dominated by reflection/scattering off cold material as assumed by the continuum model tested above. One possibility is that the $6.7 \mathrm{keV}$ line is due to reflection from highly ionized matter; we thus replaced the cold reflected (PEXRAV) power law component with an ionized reflected component, as described by the Ross \& Fabian (2005) table (otherwise known as the REFLION model). This model allows different values for the ionization parameter of the reflecting material and it also includes the $\mathrm{Fe} \mathrm{K}$ emission line, as well as emission lines from other elements in addition to the reflected continuum. We fixed the Fe abundance to be solar and we included a lower column density in front of the reflected component. The photon index of the illuminating X-ray source was kept as a free parameter. A good fit (see Fig. 2 lower panel and Table 1 model D) to the $0.5-30 \mathrm{keV}$ emission was obtained with a ionization parameter of $\xi=1000_{-430}^{+170} \mathrm{erg} \mathrm{cm} \mathrm{s}^{-1}$, where the value of the ionization was determined mainly by the strength and energy of the Fe line; at this ionization level, Fe $\mathrm{K}$ emission is almost entirely due to Fe xxv. The reflected component is modified by a lower column density absorber with $N_{\mathrm{H}} \sim 10^{22} \mathrm{~cm}^{-2}$, which is probably on a larger scale than the inner high column density absorber. We emphasize that a second highly absorbed $\left(N_{\mathrm{H}} \sim 3 \times 10^{24} \mathrm{~cm}^{-2}\right)$ power-law component is still required to account for the HXD-PIN emission and the intrinsic $2-10 \mathrm{keV}$ luminosity is $\sim 3 \times 10^{44} \mathrm{erg} \mathrm{s}^{-1}$. The photon index of the illuminating source becomes $\Gamma=1.87_{-0.28}^{+0.11}$, consistent with the mean value measured in unobscured radio quiet $\mathrm{AGN}\left(\Gamma_{\text {mean }}=1.9\right.$; Reeves \& Turner 2000).

In summary, this model is now able to reproduce in a consistent way all the main characteristic of the the broad-band X-ray emission of IRAS 19254-7245 and in particular the Fe emission line detected at $\sim 6.7 \mathrm{keV}$ and the flatness of the observed continuum. Finally, it is worth noting that, independently of the assumed model for the $0.5-10 \mathrm{keV}$ emission, we always need to include an absorbed power law component to account for the
HXD-PIN data with a $N_{\mathrm{H}} \sim 3-4 \times 10^{24} \mathrm{~cm}^{-2}$, and the derived $2-10 \mathrm{keV}$ intrinsic luminosity is always above $L(2-10 \mathrm{keV})$ $\sim 10^{44} \mathrm{erg} \mathrm{s}^{-1}$, ranging from $2 \times 10^{44} \mathrm{erg} \mathrm{s}^{-1}$ to $4 \times 10^{44} \mathrm{erg} \mathrm{s}^{-1}$ (see Table 1).

\section{Discussion and conclusions}

The detection of the Fe $\mathrm{K}$ emission line at $6.7 \mathrm{keV}$ instead of the $6.4 \mathrm{keV}$ emission line expected from neutral iron could suggest that this line is associated with strong starburst activity and that the emission below $10 \mathrm{keV}$ is not caused by the AGN, but rather by a hot thermal plasma as expected in a starburstdominated scenario. From a statistical point of view, we can obtain a good fit $\left(\chi^{2} /\right.$ d.o.f. $\left.=147 / 144\right)$ by replacing the AGN reflected emission with a thermal component. This model gives a best-fit temperature of $k T=8.1_{-1.3}^{+1.2} \mathrm{keV}, N_{\mathrm{H}} \sim 6 \times 10^{21} \mathrm{~cm}^{-2}$, twice solar abundance $\left(Z \sim 2.1 Z_{\odot}\right)$, and a luminosity of $L(2-$ $10 \mathrm{keV}) \sim 2 \times 10^{42} \mathrm{erg} \mathrm{s}^{-1}$.

A possible origin of the high temperature $(k T \sim 8 \mathrm{keV})$ plasma could be the presence of several supernovae ( $\mathrm{SNe}$ ); their $\mathrm{X}$-ray emission could in principle explain the high temperature as well as the presence of the strong $6.7 \mathrm{keV}$ line (Persic \& Rephaeli 2002). From the FIR luminosity, we estimated a star formation rate (SFR) for IRAS 19254-7245 of $\sim 200 M_{\odot} \mathrm{yr}^{-1}$

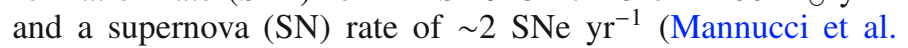
2003). However, even assuming that the $\mathrm{SNe}$ are at the higher end of the expected range of X-ray luminosity $\left(L_{\mathrm{x}}=10^{40}\right.$ $10^{41} \mathrm{erg} \mathrm{s}^{-1}$ ), a factor of 10 more $\mathrm{SNe}$ than the predicted rate would be required to maintain the observed hard X-ray emission. Furthermore, X-ray observations of starburst (SB) galaxies showed that the major contributor to the $2-10 \mathrm{keV}$ emission is the integrated emission from high-mass X-ray binaries (HMXB) and that there is a linear relation between the SFR and the 2$10 \mathrm{keV}$ emission from HMXB. In the case of IRAS 19254-7245, if all the FIR luminosity is produced by the SB activity, then the predicted 2-10 keV luminosity of the HMXB is $\sim 10^{42} \mathrm{erg} \mathrm{s}^{-1}$ (Grimm et al. 2003; Ranalli et al. 2003; Persic et al. 2004). In this scenario, we would thus require that, in contrast to other $\mathrm{SB}$, the hot diffuse emission has a luminosity similar to the contribution from HMXB. In summary, although the starburst model can well reproduce the line intensity and the overall shape of the 2-10 keV continuum, the luminosity of this thermal component $\left(L(2-10 \mathrm{keV}) \sim 2 \times 10^{42} \mathrm{rg} \mathrm{s}^{-1}\right)$ is likely to be too high to be consistent with a pure starburst scenario.

Finally, we note that if we assume that the emission detected above $2 \mathrm{keV}$ is dominated by the emission of unresolved HMXB we still cannot explain the $6.7 \mathrm{keV}$ emission line. We would expect a lower $E W$ of the Fe line $(E W \sim 0.3 \mathrm{keV}$; White et al. 1983; Persic \& Rephaeli 2002), which is inconsistent with the high value observed in IRAS 19254-7245. One possibility is that only a fraction of the Fe line at $6.7 \mathrm{keV}$ originates in a high temperature plasma. We thus included in our best-fit model a second high temperature emission fixing the abundance to the solar value. This high temperature thermal emission can account for $\sim 30 \%$ of the flux of the line at $6.7 \mathrm{keV}$. Although the inclusion of this emission can account for a fraction of the Fe line at $6.7 \mathrm{keV}$, we still need a strong ionized reflection component to account at the same time for the continuum shape and the Fe line.

On the other hand, if we attribute the hard X-ray emission to the presence of the AGN the flatness of its observed continuum together with its strong Fe emission lines are usually considered evidence of a Compton-Thick source, where no direct emission is seen below $10 \mathrm{keV}$ and the shape is produced by reflection off cold matter. However, in contrast to what observed for other 
Compton thick sources, here the Fe $\mathrm{K}$ emission is predominately in the He-like state with little or no $6.4 \mathrm{keV}$ line.

It is often assumed that a hallmark of Compton-thick X-ray sources is a prominent $\mathrm{Fe} \mathrm{K} \alpha$ emission line at $\sim 6.4 \mathrm{keV}$, from neutral matter, with an $E W$ that can exceed a keV. However, we show below that in practice, the $\mathrm{Fe} \mathrm{K} \alpha$ line $E W$ can be reduced significantly and become undetectable for large column densities of the order of $10^{25} \mathrm{~cm}^{-2}$ or more. The observed $E W$ of the Fe K line is at its largest when only the reflected continuum associated with the production of the line is observed. However, if any additional continuum is observed and if its magnitude is comparable to, or greater, than the scattered/reflected continuum, the $\mathrm{Fe} \mathrm{K}$ line will be "diluted" and the $E W$ reduced. This is because for lines of sight that intercept the reprocessor, the absolute luminosity of the $6.4 \mathrm{keV} \mathrm{Fe} \mathrm{K}$ line and its associated reflected continuum can be much less than the continuum from the Comptonthin scattering zone, so that the $E W$ of the line measured against the total observed continuum can be much less than that measured against the continuum emerging from the Compton-thick reflector.

In this scenario, the ionized Fe emission line could be produced in the same Compton-thin zone that scatters a fraction of the intrinsic continuum into the line-of-sight. If this scattered continuum is the dominant observed continuum (as would be the case if the direct line-of-sight is obscured by Compton-thick matter), then the $E W$ of the emission lines from ionized Fe could be large, of the order of hundreds of $\mathrm{eV}$ or more. The details depend on several factors, principally the ionization parameter and column density of the line-emitting region, as well as the shape of the ionizing continuum (see for example, detailed calculations of the $E W$ of the Fe XXV and Fe XXVI emission lines in Bianchi \& Matt 2002). Suppose that the Compton-thick reprocessor subtends a solid angle $\Delta \Omega / 4 \pi$ at the X-ray source and that the system is observed along a line-of-sight that does not provide a direct view of the X-ray source and has the greatest column density, $N_{H}$, (to maximize the Fe $\mathrm{K}$ line $E W$ ). Further suppose that the space between the reprocessor is filled by a warm, optically-thin scattering zone with Thomson depth $\tau_{\text {thin }}$ (with $\left.\tau_{\text {thin }} \ll 1\right)$, subtending a solid angle $1-(\Delta \Omega / 4 \pi)$ at the $\mathrm{X}$-ray source. Then, a fraction $f \equiv \tau_{\text {thin }}[1-(\Delta \Omega / 4 \pi)]$ of the intrinsic X-ray continuum is scattered into the observer's lineof-sight and will reduce the $E W$ of the Fe $\mathrm{K}$ line if it dominates over the zeroth-order continuum that is observed directly through the Compton-thick absorber. Specifically, for column densities greater than a few $\times 10^{24} \mathrm{~cm}^{-2}$, even a small value of $f$ can significantly reduce the $E W$ of the $\mathrm{Fe} \mathrm{K}$ line. For example, for a column density of $10^{25} \mathrm{~cm}^{-2}$, a scattering fraction of $f>0.01$ will reduce the $E W$ of an Fe $\mathrm{K}$ line by more than an order of magnitude, so that an $E W$ of $1 \mathrm{keV}$ would be reduced to less than $\sim 100 \mathrm{eV}$, and it could render the line undetectable (see Ghisellini et al. 1994).

Furthermore, the intrinsic $E W$ (i.e., prior to the dilution effect) of Fe K lines depends on several factors, not only the column density of the absorber but also the geometry of the absorber (e.g., the half opening angle of the putative torus; see Ghisellini et al. 1994; Ikeda et al. 2009; Matt et al. 1996). For example, for an half opening angle of $30^{\circ}$ and our estimate of the column density of the neutral absorber, the intrinsic $E W$ of the $6.4 \mathrm{keV}$ Fe line can span the range from 1 to $4 \mathrm{keV}$ (Ghisellini et al. 1994).

The inferred column density of the Compton-thick reprocessor implies that a scattering fraction of only $\sim 0.1 \%$ in the optically-thin zone is required to begin to dilute the $6.4 \mathrm{keV}$ $\mathrm{Fe} \mathrm{K}$ line and a scattering fraction of a few percent is sufficient to reduce the $E W$ of the line well below $100 \mathrm{eV}$, consistent with the upper limit to the $E W$ and the $\sim 2 \%$ scattering fraction (measured with respect to the de-absorbed primary power law component) as measured with the Suzaku data.

A second possible geometry is that we have a direct view of the inner surface of the Compton-thick reprocessor, but the outer part of this reprocessor is ionized. In this case, if the remaining part of the reprocessor is Compton-thin, the $E W$ of the $6.4 \mathrm{keV} \mathrm{Fe} \mathrm{K} \alpha$ line will be reduced and the emission detected below $10 \mathrm{keV}$ is the reflected emission from this inner ionized surface of the torus, which will also produce a strong $6.7 \mathrm{keV}$ line. Thus, we see that the lack of a large $E W$ neutral Fe $\mathrm{K}$ in IRAS 19254-7245 is not unexpected. The spectrum below $10 \mathrm{keV}$ is then dominated by this optically-thin scattered continuum and the dominance of the emission line from ionized Fe is consistent with this picture.

We note that this is not a unique case of a detection of a strong $6.7 \mathrm{keV}$ line in a luminous infrared galaxy. Other examples are Arp299 (Ballo et al. 2004), Arp220 (Iwasawa et al. 2005; Teng et al. 2009), and IRAS 00182-7112 (Nandra \& Iwasawa 2007). For all these sources although the optical spectra show no clear signature of AGN activity, their X-ray emission and the $6.7 \mathrm{keV}$ line can be explained by the presence of an AGN and an ionized reflector as in the case of IRAS 192547245. However, while in the case of Arp 299 and Arp 220, the X-ray luminosity is not indicative of the major contributor to the bolometric luminosity being a high-luminosity AGN, in the case of IRAS 00182-7112 the X-ray luminosity is too high to be accounted for by the strong starforming activity (L(210) $>10^{44} \mathrm{erg} \mathrm{s}^{-1}$ ) as for IRAS 19254-7245. For all these sources, the presence of a strong ionized Fe line, with little or no $6.4 \mathrm{keV}$ line, could be reconciled with the picture of a heavily obscured AGN assuming that we do not have a direct view of the reflected continuum; this is because the continuum is diluted by the scattering from the ionized matter, which is associated with the production of the line, or if the surface of the putative Compton-thick reprocessor is highly ionized.

Overall to account for the X-ray emission of IRAS 192547245 above $2 \mathrm{keV}$, we need two absorbing/reflecting media: one neutral and Compton-thick and probably seen in transmission, and one ionized and seen probably in reflection. This latter is responsible for the flat X-ray spectrum emerging below $10 \mathrm{keV}$, for the He-like Fe $\mathrm{K}$ line and probably for the dilution of the $6.4 \mathrm{keV}$ Fe line produced in the neutral Compton-thick absorber.

Despite the various possible models for the $0.5-10 \mathrm{keV}$ emission, we always need a neutral high column density absorber to account for the emission emerging above $10 \mathrm{keV}$. Once we have corrected for the amount of absorption, the intrinsic 2-10 keV luminosity of the primary AGN component is $\sim 10^{44} \mathrm{erg} \mathrm{s}^{-1}$. This high column density absorber may also be the one responsible for the deep hydrocarbon absorption detected in the $L$-band spectrum (Risaliti et al. 2003) at $3.4 \mu \mathrm{m}$. Finally, independently of the assumed model for the $2-35 \mathrm{keV}$ emission (i.e., ionized reflection or scattered power-law component), we always require the soft thermal component to account for the $0.5-2 \mathrm{keV}$ emission. As already found for other ULIRGs, this thermal component has a temperature $k T \sim 0.7 \mathrm{keV}$, and the luminosity of this component is $L(0.5-2 \mathrm{keV}) \sim 4 \times 10^{41} \mathrm{erg} \mathrm{s}^{-1}$. Although, we cannot exclude a possible contribution from the ionized reflector, the measured soft X-ray luminosity agrees with that expected from the FIR luminosity and the SFR of IRAS 19254-7245 (Ranalli et al. 2003; Persic et al. 2004).

The total X-ray luminosity estimated from the intrinsic component at $E>10 \mathrm{keV}$ can be converted into a bolometric 
luminosity and compared with the total infrared emission. We adopted the $\alpha_{O X}$-luminosity correlation of Steffen et al. (2006) to estimate the $2500 \AA$ luminosity, and the Elvis et al. (1994) quasar spectral energy distribution to estimate the total luminosity of the AGN component. Assuming $L_{2-10} \sim 10^{44} \mathrm{erg} \mathrm{s}^{-1}$, we obtained $L_{\mathrm{BOL}}(A G N) \sim 2 \times 10^{45} \mathrm{erg} \mathrm{s}^{-1}$, i.e., about $50 \%$ of the infrared luminosity. A different way of estimating the AGN luminosity is from its emission in the mid-infrared, the only other band where the continuum emission of the AGN is not completely absorbed. From an analysis of the Spitzer-IRS spectrum, Nardini et al. (2008) estimated an AGN contribution to the bolometric luminosity of IRAS $19254-7245$ of $25 \%$. Considering the uncertainties in the bolometric corrections in both the X-ray and the mid-infrared bands, the two estimates can be considered to be in rough agreement. If the difference is assumed to be real, this could be an indication of the non-complete covering factor of the AGN circumnuclear absorber: indeed, the estimate from the mid-infrared spectrum is done assuming a complete reprocessing of the intrinsic AGN emission in the infrared. However, the optical classification of IRAS $19254-7245$ as a Seyfert 2 suggests that the obscuration of the nuclear source is not complete. The estimates from the X-ray and infrared spectra could then be perfectly reconciled by assuming a covering factor of the obscuring material of about $50 \%$.

In conclusion, this deep Suzaku observation has allowed us to measure for the first time the hard X-ray emission of IRAS 19254-7245 and infer that its intrinsic 2-10 keV luminosity is about few times $10^{44} \mathrm{erg} \mathrm{s}^{-1}$. We have found evidence that the AGN hosted in IRAS 19254-7245 is highly obscured, with a measured column density of the neutral absorber of $N_{\mathrm{H}} \sim 3 \times 10^{24} \mathrm{~cm}^{-2}$. We confirm the presence of a strong iron $\mathrm{K}$ emission line with an $E W \sim 0.7 \mathrm{keV}$. The energy of iron $\mathrm{K}$ emission line is found to be consistent with Fe XXV. We propose that the X-ray emission detected below $10 \mathrm{keV}$ can be ascribed to scattered/reflected emission from highly ionized matter, which could be identified with the warm Compton-thin gas that fills the space between the neutral Compton-thick reprocessor.

Acknowledgements. V.B. acknowledge support from the UK STFC research council. R.D.C. acknowledge financial support from the ASI (Agenzia Spaziale Italiana) grant $\mathrm{I} / 088 / 06 / 0$. Support for this work was provided by the National Aeronautics and Space Administration through the NASA grant NNG04GB78A. We thank the anonymous referee for his/her useful comments, which have improved this paper.

\section{References}

Ballo, L., Braito, V., Della Ceca, R., et al. 2004, ApJ, 600, 634 Bianchi, S., \& Matt, G. 2002, A\&A, 387, 76

Boldt, E. 1987, Phys. Rep., 146, 215

Braito, V., Franceschini, A., Della Ceca, R., et al. 2003, A\&A, 398, 107 Braito, V., Della Ceca, R., Piconcelli, E., et al. 2004, A\&A, 420, 79 Caccianiga, A.,Severgnini, P., Braito, V., et al. 2004, A\&A, 416, 901 Caccianiga, A., Severgnini, P., Della Ceca, R., et al. 2008, A\&A, 477, 735 Della Ceca, R., Maccacaro, T., Caccianiga, A., et al. 2004, A\&A, 428, 383 Elvis, M., Wilkes, Belinda J., McDowell, J. C., et al. 1994, ApJS, 95, 1 Franceschini, A., Braito, V., Persic, M., et al. 2003, MNRAS, 343, 1181 Frontera, F., Orlandini, M., Landi, R., et al. 2007, ApJ, 666, 86 Fukazawa, Y., Mizuno, T., Watanabe, S., et al. 2009, PASJ, 61, 17 Genzel, R., Lutz, D., Sturm, E., et al. 1998, ApJ, 498, 579

Ghisellini, G., Haardt, F., \& Matt, G. 1994, MNRAS, 267, 743

Grimm, H.-J., Gilfanov, M., \& Sunyaev, R. 2003, MNRAS, 339, 793

Gruber, D. E., Matteson, J. L., Peterson, L. E., \& Jung, G. V. 1999, ApJ, 520, 124

Kokubun, M., Makishima, K., Takahashi, T., et al. 2007, PASJ, 59, 53

Hopkins, P. F., Hernquist, L., Cox, T. J., et al. 2005, ApJ, 630, 705

Hopkins, P. F., Hernquist, L., Cox, T. J., et al. 2006, ApJS, 163, 1

Ikeda, S., Awaki, H., \& Terashima, Y. 2009, ApJ, 692, 608

Imanishi, M., Terashima, Y., Anabuki, N., \& Nakagawa, T. 2003, ApJ, 596, L167

Iwasawa, K., Sanders, D. B., Evans, A. S., et al. 2005, MNRAS, 357, 565

Koyama, K., Tsunemi, H., Dotani, T., et al. 2007, PASJ, 59, 23

Magdziarz, P., \& Zdziarski, A. A. 1995, MNRAS, 273, 837

Mannucci, F., Maiolino, R., Cresci, G., et al. 2003, A\&A, 401, 519

Matt, G., Brandt, W. N., \& Fabian, A. C. 1996, MNRAS, 280, 823

Mitsuda, K., Bautz, M., Inoue, H., et al. 2007, PASJ, 59, 1

Moretti, A., Pagani, C., Cusumano, G., et al. 2009, A\&A, 493, 501

Nandra, K., \& Iwasawa, K. 2007, MNRAS, 382, L1

Nardini, E., Risaliti, G., Salvati, M., et al. 2008, MNRAS, 385, L130

Page, K. L., Reeves, J. N., O’Brien, P. T., Turner, M. J. L., \& Worrall, D. M. 2004, MNRAS, 353, 133

Persic, M., \& Rephaeli, Y. 2002, A\&A, 382, 843

Persic, M., Rephaeli, Y., Braito, V., et al. 2004, A\&A, 419, 849

Ptak, A., Heckman, T., Levenson, N. A., Weaver, K., \& Strickland, D. 2003, ApJ, 592,782

Ranalli, P., Comastri, A., \& Setti, G. 2003, A\&A, 399, 39

Reeves, J. N., \& Turner, M. J. L. 2000, MNRAS, 316, 234

Risaliti, G., Maiolino, R., Marconi, A., et al. 2003, ApJ, 595, L17

Ross, R. R., \& Fabian, A. C. 2005, MNRAS, 358, 211

Sanders, D. B., \& Mirabel, I. F. 1996, ARA\&A, 34, 749

Springel, V., Di Matteo, T., \& Hernquist, L. 2005, MNRAS, 361, 776

Steffen, A. T., Strateva, I., Brandt, W. N., et al. 2006, AJ, 131, 2826

Takahashi, T., Abe, K., Endo, M., et al. 2007, PASJ, 59, 35

Teng, S. H., Wilson, A. S., Veilleux, S., et al. 2005, ApJ, 633, 664

Teng, S. H., Veilleux, S., Anabuki, N., et al. 2009, ApJ, 691, 261

Vignati, P., Molendi, S., Matt, G., et al. 1999, A\&A, 349, L57

Weedman, D. W., \& Houck, J. R. 2008, ApJ, 686, 127

White, N. E., Swank, J. H., \& Holt, S. S. 1983, ApJ, 270, 711

Wilms, J., Allen, A., \& McCray, R. 2000, ApJ, 542, 914

Yaqoob, T. 1997, ApJ, 479, 184 